\title{
INITIAL MOTION OF A RECTANGULAR OBJECT BEING PUSHED OR PULLED
}

D.T.Pham and K.C.Cheung

University of Wales (Cardiff), P.0.Box 904,

Cardiff, CPI $3 \mathrm{YH}$, U.K.
S.H.Yeo

University of Birmingham, P.0.Box 363,

Birmingham, B15 2TT, U.K.

\begin{abstract}
This paper describes techniques for determining the location of the initial centre of rotation (COR) of a rectangular bar being pushed or pulled. The initial $C O R$ is the point about which the bar first rotates when the pushing or pulling force is applied. This point characterises the initial motion of the bar. The paper also investigates how the location of the initial COR varies with the magnitude of the exerted force. The results obtained are to be used in the design of materials handling machines in which workpieces are manipulated by pushing or pulling to save the time picking them up and transferring them to their delivery point.
\end{abstract}

\section{Introduction}

Discrete materials handling is often performed by pushing or pulling the components to be handled without grasping them. Bxamples abound in high-speed packaging where the packages to be handled (such as cigarette cartons or confectionery bars) cannot be manipulated by pick-and-place operations due to lack of time. This paper forms part of a study into how components move when pushed or pulled. Previous work on this topic includes quasi-static analysis of pushing, modelling of the dynamics of impact and numerical simulation of the motion of objects being pulled. Mason [1-3] obtained fundamental results giving the sense and centre of rotation of a pushed object during quasi-static pushing. Peshkin [4] and Peshkin and Sanderson [5-7] also investigated quasi-static pushing and developed manipulation strategies based on their findings. Wang and Mason [8] and Wang [9] studied the dynamics of impact where inertia forces dominate frictional forces. By numerically simulating the motion of a rectangular bar under traction, Pham and Cheung [10,11] addressed the case where neither frictional forces nor inertial forces dominate.

This paper presents methods to determine the location of the initial centre of rotation (COR) of a rectangular bar being pushed or pulled. The initial COR is the point about which the bar first rotates when the pushing or pulling force is applied. This point characterises the initial motion of the bar. The paper also investigates how the location of the initial COR varies with the magnitude and point of application of the exerted force.

\section{Methods of Finding Initial Centre of Rotation for Different Categories of Motion}

\subsection{Quasi-static Motion}

When the inertial forces on a moving object are negligible, the motion is known as quasi-static.

The aim of this section is to find the initial instantaneous centre of rotation of an object resting on a horizontal support plane when pushed or pulled by a force. A method of doing this is to take moments about the point of application of the force and then equating the moment to zero as inertial forces are negligible [12]. In this section a different approach is employed to find the centre of rotation.

Consider the rectangular bar in Figure 1 . Let $R$ be the distance between the centre of rotation COR and the centre of mass $C M$ and $y_{n}$ be the distance between $C M$ and the point of application $P$ of pulling force $F$.

Let $M_{c}$ be the frictional moment about the COR. $M_{c}$ is a function of $R$. Taking moments about COR, for motion to be possible:

$$
P\left(R+y_{n}\right)-M_{c}>0
$$

In the quasi-static case, $F\left(R+y_{n}\right)-M_{c}=0$

$$
P=\frac{M_{c}}{R+y_{n}}
$$

For given $y_{n}^{\prime}$, the $C O R$ will be so located as to minimize the value of $P$ that can cause rotation. This is termed the minimum effort criterion.

By differentiating $P$ with respect to $R$ and equating the derivative to zero, $a$ value of $R$ can be found which defines the centre of rotation for 
quasi-static motion (or simply, the "quasi-static" COR). his approach to finding the quasi-static COR will be shown to yield the same result as that adopted by Mason [12].

\section{Method}

Por the rectangular bar shown in Figure 1 , the friction moment about point 0 , the COR, caused by the rotation of an element is:

$$
\begin{aligned}
d M_{c} & =d f \times \rho \\
& =\frac{-\mu \mathrm{mg}}{4 \mathrm{ab}} \operatorname{sgn}(\dot{\theta}) \rho \mathrm{d} \rho \mathrm{d} \gamma \times \rho
\end{aligned}
$$

where $m$ is the mass and $\dot{\theta}$ the angular velocity of the object and $\mu$ the friction coefficient.

$$
\begin{aligned}
d M_{c} & =\frac{-\mu \mathrm{mg}}{4 \mathrm{ab}} \operatorname{sgn}(\dot{\theta}) \rho^{2} \mathrm{~d} \rho d \gamma \\
M_{c} & =\frac{-\mu \mathrm{mg}}{4 \mathrm{ab}} \operatorname{sgn}(\dot{\theta}) \iint_{\mathrm{B}} \rho^{2} \mathrm{~d} \rho \mathrm{d} \gamma
\end{aligned}
$$

After integrating:

$\iint_{s} \rho^{2} \mathrm{~d} \rho \mathrm{d} \gamma$

$$
\begin{aligned}
& =\left(-h_{1} h_{2} \sqrt{h_{1}^{2}+h_{2}^{2}}+h_{2} h_{3} \sqrt{h_{2}^{2}+h_{3}^{2}}-h_{3} h_{4} \sqrt{h_{3}^{2}+h_{4}^{2}}\right. \\
& \left.+h_{4} h_{1} \sqrt{h_{4}^{2}+h_{1}^{2}}\right) / 3+\frac{1}{6}\left[h_{1}^{3} 1 n\left|\frac{\sqrt{h_{1}^{2}+h_{2}^{2}}-h_{2}}{\sqrt{h_{4}^{2}+h_{1}^{2}}-h_{4}}\right|\right. \\
& +h_{2}^{3} l_{\frac{1 n}{h_{2}^{2}+h_{3}^{2}}+h_{3}}\left|+h_{3}^{3} 1 n\right| \sqrt{\frac{h_{3}^{2}+h_{4}^{2}}{h_{1}^{2}+h_{2}^{2}}+h_{1}}\left|\sqrt{h_{2}^{2}+h_{3}^{2}}-h_{2}\right| \\
& \left.+h_{4}^{3} \ln \left|\frac{\sqrt{h_{4}^{2}+h_{1}^{2}}+h_{1}}{\sqrt{h_{2}^{2}+h_{3}^{2}}+h_{3}}\right|\right]
\end{aligned}
$$

where $h_{1}=R \sin \alpha-b, h_{2}=R \cos \alpha+a, h_{3}=R \sin \alpha+b$, $h_{4}=R \cos \alpha-a$. ( $R$ is the distance between $C M$ and COR; $\alpha$ is the inclination of the line joining $C M$ and $C O R$ with the horizontal axis)

As the applied force is along the $x$-axis, there is no impending motion in the $y$-direction, hence no component of friction force in the $y$-direction. So the initial motion will be translation along the $\mathrm{x}$-direction with anti-clockwise rotation. Therefore the initial COR lies on the axis through the $C M$ of the object and parallel to $y$. Hence $\alpha=270$.

Substituting this value of $\alpha$ into the expressions for $h_{1}, h_{2}, h_{3}$ and $h_{4}$ yields:

$$
h_{1}=-(R+b), h_{2}=a, h_{3}=-(R-b), h_{4}=-a \text {. }
$$

M can then be written as

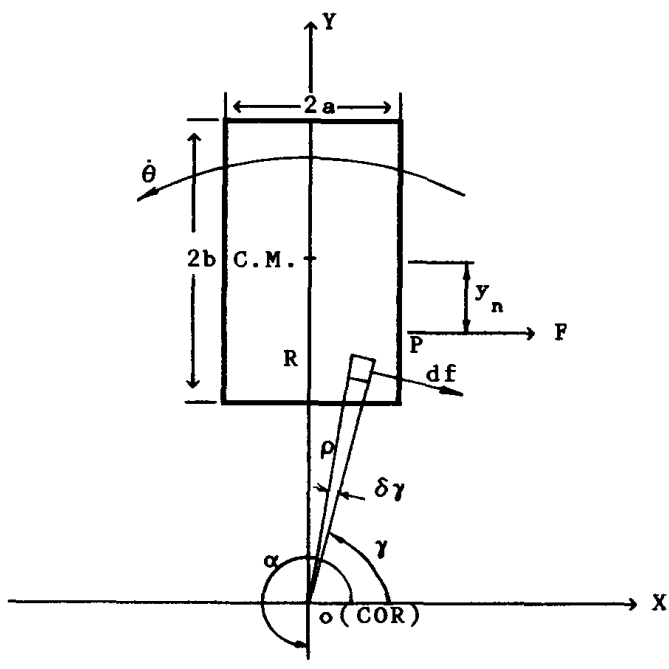

Figure 1 Friction moment about COR of a rectangular bar

$$
M_{c}=\frac{-\mu m g}{4 a b} \operatorname{sgn}(\dot{\theta}) \times g_{1}(R)
$$

where

$$
\begin{aligned}
& g_{1}(R) \\
& \begin{aligned}
=(2 a / 3) & \left((R+b) \sqrt{R^{2}+a^{2}+b^{2}+2 R b}-(R-b) \sqrt{R^{2}+a^{2}+b^{2}-2 R b}\right) \\
& +\frac{1}{6}\left[2 a^{3} \ln \left|\frac{\sqrt{R^{2}+a^{2}+b^{2}-2 R b}-R+b}{\sqrt{R^{2}+a^{2}+b^{2}+2 R b}-R-b}\right|\right. \\
& -(R+b)^{3} \ln \left|\frac{\sqrt{R^{2}+a^{2}+b^{2}+2 R b}-a}{\sqrt{R^{2}+a^{2}+b^{2}+2 R b}+a}\right| \\
& \left.-(R-b)^{3} \ln \left|\frac{\sqrt{R^{2}+a^{2}+b^{2}-2 R b}+a}{\sqrt{R^{2}+a^{2}+b^{2}-2 R b}-a}\right|\right]
\end{aligned}
\end{aligned}
$$

From Eq (1)

$$
\mathrm{F}=\frac{\frac{-\mu \mathrm{mg}}{4 \mathrm{ab}} \operatorname{sgn}(\dot{\theta}) \times g_{1}(R)}{R+y_{n}}
$$

$\frac{d F}{d R}$

$=\frac{-\mu m g}{4 a b} \operatorname{sgn}(\dot{\theta})\left[\left(R+y_{n}\right) \frac{d g_{1}(R)}{d R}-g_{1}(R)\right] /\left(R+y_{n}\right)^{2}$

Putting $\frac{d P}{d R}=0$ gives 


$$
\left(R+y_{n}\right) \frac{d g_{1}(R)}{d R}-g_{1}(R)=0
$$

Solving Eq (2) will yield the $R$ that minimizes $P$.

This is a one-dimensional root finding problem. It is solved numerically using the van Wijngaarden-Dekker-Brent Method [13]. This method combines root bracketing, bisection and inverse quadratic interpolation to converge from the neighbourhood of a zero crossing to the actual root. Therefore it possesses the sureness of bisection with the speed of a higher order method when appropriate.

The results obtained for an arbitrarily sized object $(390 \mathrm{~mm} \times 40 \mathrm{~mm})$ are shown in Table 1 .

Table 1 Values of $R_{\text {quasi }}$ obtained using the minimum effort criterion

\begin{tabular}{|l|l|}
\hline$y_{n}(m)$ & $R_{q u a s i}(m)$ \\
\hline 0.01 & 0.18920 \\
0.05 & 0.15308 \\
0.10 & 0.12057 \\
0.15 & 0.09723 \\
0.19 & 0.08334 \\
\hline
\end{tabular}

Table 2 shows the values of $R$ for the quasi-static case, $R_{\text {quas }}$, obtained using Mason's zero-moment approach which is described in [14].

Table 2 Values of $R_{\text {qua }}$ obtained using Mason's zero-moment approach

\begin{tabular}{|l|l|}
\hline$y_{n}(\mathrm{~m})$ & $\mathrm{R}_{\mathrm{quas}}(\mathrm{m})$ \\
\hline 0.01 & 0.18920 \\
0.05 & 0.15308 \\
0.10 & 0.12057 \\
0.15 & 0.09723 \\
0.19 & 0.08334 \\
\hline
\end{tabular}

It can be seen that the location of the quasi-static COR as found when applying the minimum effort criterion is the same as that predicted by Mason's technique.

\subsection{Impulsive motion}

When the inertial forces of a moving object dominate over the frictional forces, the motion is referred to as impulsive. It is known [15] that if $P$ is an impulse then the following relationship holds:

$$
L=\left(k_{G}^{2}+R_{i m p u l}^{2}\right) / R_{\text {impul }}
$$

where $R_{\text {inpul }}$ is the value of $R$ for the case of impulsive motion, $k_{G}$ is the radius of gyration of the object being hit by $F$ and $L$ the distance between the point of application of $F$ and the point about which the object will instantaneously rotate (i.e. the "impulsive" COR) (Pigure 2).

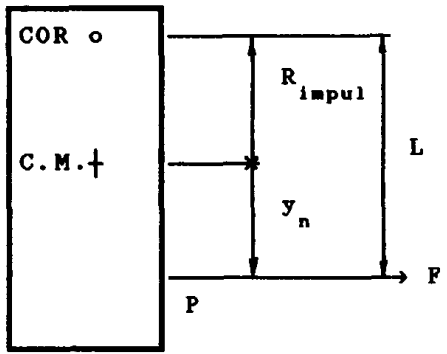

Figure 2 Definition of geometrical parameters

Note that Eq (3) also gives the initial COR for the case when $P$ is $a$ step function and frictional resistance is negligible.

Relative to $\mathrm{CM}$, the location of the initial (impulsive) COR is defined by

since $L=R_{i \text { mpul }}=k_{G}^{2} / y_{n}$

impul $n$

Clearly, if the bar is hinged at the COR thus determined, point $P$ will be the centre of percussion well know $n$ in mechanics [15].

\subsection{Dynamic motion}

This problem lies between the quasi-static and impulsive motion problems. Here, the applied force $P$ is sufficiently high to accelerate the object being pushed, but is still below the level beyond which friction effects can be ignored.

The initial COR was obtained by performing the numerical integration described in $[10,11]$ for a small time interval, typically $10^{-5}$ second. As expected, it was found that for a given point of application of $F$, the COR lay between the corresponding quasi-static and impulsive centres as shown in Figure 3.

3.Variation of quasi-static centre and impulsive centre with point of application of force

As seen from Figure 3, both the quasi-static and impulsive $C O R$ are functions of $y_{n}$,the distance between the centre of mass $C M$ and the point of application of $P$. Por small values of $y_{n}$, i.e. when $P$ is applied near to $C M$, the impulsive COR is further away from $C M$ than its quasi-static counterpart (Figure 4a). 


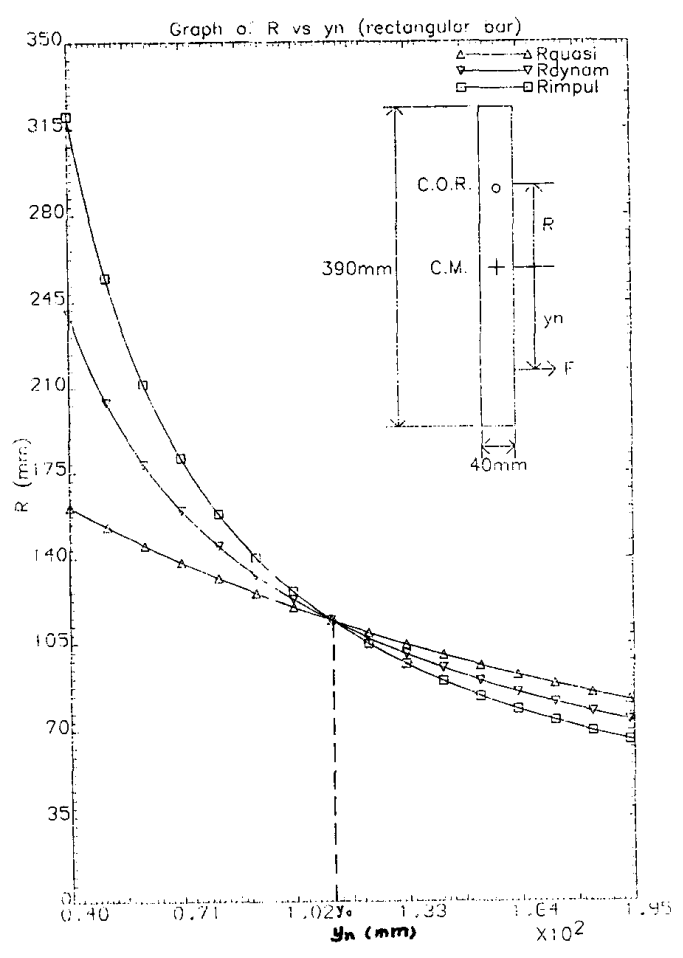

Pigure 3 Variation of $R$ with $y_{n}$ for a rectangular bar

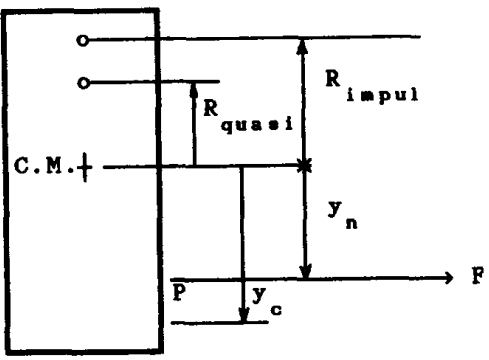

Pigure $4 a$ Relative magnitude of $R$ quasi ${ }^{\text {and }}$
$R_{i m p u l}$ for $y_{n}<y_{c}$

As $y_{n}$ increases, both CORs move towards CM. The rate of displacement is higher for the impulsive COR than that for the quasi-static COR.

At point $y_{n}=y_{c}$, the two CORs become coincident.

For $y_{n}>y_{c}$, the impulsive $C O R$ is nearer to $C M$ than the quasi-static COR (Figure 4b).

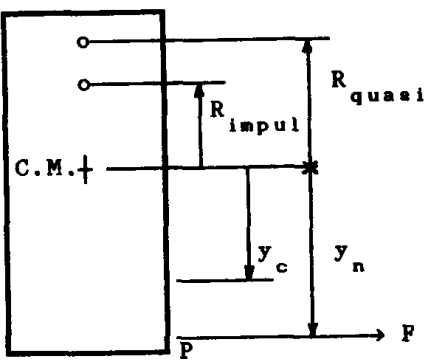

Pigure 4b Relative magnitude of $R_{q u a s}$ and $R_{i n p u l}$ for $y_{n}>_{c}$

4.Results

\subsection{Motion on Prictionless Support}

In this case there is no quasi-static COR. The object will rotate about the impulsive COR independently of the magnitude of the applied force.

\subsection{Motion on Prictional Support}

\subsubsection{Combined Bffects of Magnitude and Point of Application of Force}

When the magnitude of the applied force $F$ is 8 mall, the initial COR lies near to the quasi-static COR. As the magnitude of $F$ increases, depending on the point of application of $P$, the initial COR will either move away from $C M$ or move towards it.

When the point of application of $F$ is near $C M$ (i.e. when $y_{n}$ is small), the initial COR moves away from $C M$ as the magnitude of $F$ increases. When the point of application of $F$ is near the end of the object (i.e. when $y_{n}$ is large), the initial COR moves towards $C M$ as the magnitude of $F$ increases.

The reason for this phenomenon resides in the fact that as $P$ increases, the initial COR moves from the quasi-static COR towards the impulsive COR. As seen from Figure 3, for small values of $y_{n}$ the impulsive COR lies further away from $C M$ than the quasi-static COR. Therefore, when the magnitude of $P$ increases, the initial $C O R$ moves away from $C M$. For large values of $y_{n}$, the impulsive $C O R$ lies nearer to $C M$ than the quasi-static COR. Therefore, as the magnitude of $P$ increases, the initial COR moves towards $\mathrm{CM}$.

\subsubsection{Special Case When the Quasi-static and Impulsive Centres Coincide}

It can be seen from Pigure 3 that there exists a $y_{c}$ such that the quasi-static and impulsive centres of rotation coincide. 
Method of finding $y_{c}$

From Eqs (2) and (4), it can be seen that $R_{\text {quas }}$ and $R_{i m p u l}$ are functions of $y_{n}$ only. (Although $\mathrm{Eq}(2)$ is a polynomial equation, it can be rearranged into the form $R_{\text {quasi }}=f_{R}\left(y_{n}\right)$ where $f_{R}$ is a function of $y_{n}$.)

It follows that the difference between $R_{\text {quasi }}$ and $R_{i m p u l}$ is also a function of $y_{n}$.

The function $R_{\text {diff }}\left(y_{n}\right)=R_{\text {quasi }}\left(y_{n}\right)-R_{\text {impul }}\left(y_{n}\right)$ is constructed and the Van Wijngaarden-Dekker-Brent Method is again used to solve for $y_{n}$ such that $R_{\text {diff }}=0$. It was found that $y_{c}=112.23 \mathrm{~mm}$ and the corresponding $R$ is $114.11 \mathrm{~mm}$.

The significance of this result is that when the object is being pulled at the point thus determined, regardless of the magnitude of the applied force (provided that the latter is at least equal to the magnitude required to cause motion), it will always start to rotate about a known point which can be determined without using either the Direct Integration method or the Predictor-Corrector search described in $[10,11]$.

\section{Conclusion}

The minimum effort criterion has been proved to be able to predict the quasi-static centre of rotation.

It has been found that the initial COR always lies between the quasi-static and the impulsive CORs and that it will move towards the impulsive COR as the magnitude of the applied force increases.

There exists a point on an object such that when the force is applied at that point, the object will start to rotate about a known point.

Note that although the analysis reported here has been carried out for an object under traction, the results apply equally well to the case where the object is being pushed.

\section{Acknowledgements}

The authors would like to thank the following organisations for supporting this work: the Science and Engineering Research Council (Specially Promoted Programme on High Speed Machinery). Cadbury Schweppes plc., the Croucher Foundation, and the Committee of Vice Chancellors and Principals (ORS award programme). They would also like to thank Mr. T.W.Greeves of Cambridge Consultants (Systems Bngineering) Ltd for his help.

\section{References}

[1] Mason, M.T. Mechanics of pushing. Proc. $2^{\text {nd }}$ International Symposium on Robotics Research, 1984, H.Hanafusa and H.Inoue eds, MTT Press, P p.421-428

[2] Mason, M.T. On the scope of quasi-static pushing. Robotics Research: The Third International Symposium, 1986, 0.D.Faugeras and G.Giralt eds. MIT Press, pp.229-233

[3] Mason, M.T. Mechanics and planning of manipulator pushing operations. The International Journal of Robotics Research, Vol.5, No.3, 1986, P P.53-71

[4] Peshkin, M.A. Planning robotic manipulation strategies for sliding objects. Ph.D. Thesis, Department of Physics, The Robotics Institute, Carnegie Mellon University, Pittsburgh, Pennsylvania, USA, November 1986

[5] Peshkin, M.A. and Sanderson, A.C. Planning sensorless robot manipulation of sliding objects. AAAI-86 National Conference on Artificial Intelligence, 1986, American Association for Artificial Intelligence, pp.1107-1112

[6] Peshkin, M.A. and Sanderson, A.C. Planning robotic manipulation strategies for workpieces that slide. IERE Journal of Robotics and Automation, Vol.4, No.5, October 1988, pp.524-531

[7] Peshkin, M.A. and Sanderson, A.C. The motion of a pushed, sliding workpiece. IEEE Journal of Robotics and Automation, Vol.4, No.6, December 1988, p p.569-598

[8] Wang, Y. and Mason, M.T. Modelling impact dynamics for robotic operations. Proc. 1987 IEBE International Conference on Robotics and Automation, Vol.2, March 31-April 3, 1987, Raleigh, North Carolina, USA, pp.678-685

[9] Wang, Y. On impact dynamics of robotic operations. Carnegie Mellon University Robotics Institute Technical Report CMU-RI-TR-86-14, September 1986

[10] Pham, D.T. and Cheung, K.C. Motion of a rectangular bar under traction on a horizontal plane. Proc. $7^{\text {th }}$ International Conference on Mathematical and Computer Modeling, 2-5 August, 1989, Chicago, USA

[11] Pham, D.T. and Cheung, K.C. Dynamics of a bar under traction. Submitted to IBEB Transactions on Robotics and Automation. (August 1989)

[12] Mason, M.T. and Salisbury, J.K. Robot hands and the mechanics of manipulation. MIT Press 1985 , Section II Chapter 2

[13] Brent, R.D. Algorithms for minimisation without derivatives. Englenood Cliffs, Nev Jersey, Prentice Hall, 1973, Chapters 3,4

[14] Pham, D.T. and Cheung, K.C. Determination of the initial centre of rotation of a bar being pushed or pulled during handling. Internal Report, Automation and Robotics Centre, University of Wales, Cardiff, U.K., 1990

[15] Routh, B.J. The elementary part of a treatise on the dynamics of a system of rigid bodies. $7^{\text {th }}$ edition, Dover Publications, New York 